\title{
Revealing the Thermodynamic Background of the Memory Effect in Phase Separating Cathode Materials
}

\author{
Klemen Zelič1 - Igor Mele1 - Ivo Pačnik ${ }^{1}$ - Jože Moškon ${ }^{2}$ - Miran Gaberšček ${ }^{2}$ - Tomaž Katrašnik ${ }^{1, *}$ \\ 1University of Ljubljana, Faculty of Mechanical Engineering, Slovenia \\ 2National Institute of Chemistry, Slovenia
}

\begin{abstract}
Phase separating Li-ion battery cell cathode materials feature a well-known phenomenon called the memory effect. It manifests itself as an abnormal change in working voltage being dependent on cell cycling history. It was only recently that plausible mechanistic reasoning of the memory effect in Li-ion batteries was proposed. However, the existing literature does still not consistently reveal a phenomenological background for the onset or absence of the memory effect. This paper provides strong experimental and theoretical evidence of the memory effect in phase separating Li-ion battery cathode materials. Specifically, the background leading to the onset or absence of the memory effect and the underlying causal chain of phenomena from the collective particle-by-particle intra-electrode phenomena to macroscopic voltage output of the battery are presented and discussed. The results, clearly reveal that no memory effect is observed and predicted for low cut off voltages, whereas the absence of the first rest in memory writing cycle does not result in the absence of the memory effect, as previously believed. In addition, excellent agreement between the simulated and measured results is shown which, on one hand confirms the credibility of the combined analyses and, on the other, provides clear causal relations from macroscopic experimental parameters to simulated phenomena on the particle level.
\end{abstract}

Keywords: Li-ion battery, phase separating material, multi-particle porous electrode, memory effect

Highlights

- Innovative evidence on the onset and absence of the memory effect in phase separating cathode materials is provided.

- Analysis is based on combining experimental results and advanced continuum modelling on porous battery electrodes.

- Memory effect is not present if the cut off voltage in memory writing cycle is sufficiently low.

- The absence of first rest in memory writing cycle still leads to the intra-particle distribution of lithium yielding a memory effect .

\section{INTRODUCTION}

Batteries are one of the most widespread energy storage devices, covering a very broad range of mobile, and increasingly also stationary applications. They are thus enablers for more sustainable mobility and more user-friendly leisure applications. With the introduction of renewable energy sources, they are gaining on significance in energy applications.

In battery applications, one of the most important control parameters is the so called state-of-charge (SOC) representing the level of charge of an electric battery relative to its capacity. In most applications SOC estimation is performed via voltage reading. For batteries composed of layered materials, e.g. NMC, $\mathrm{LiCoO}_{2}, \mathrm{LiMnO}_{2}, \mathrm{LiNiO}_{2}$, that are not subjected to phase transitions and are thus characterized by a relatively steep voltage gradient during the changing of the lithiation level of electrode [1], such a procedure is less demanding. By contrast, SOC estimation is much more demanding for batteries composed of olivine materials featuring a very flat voltage profile between spinodal points of the chemical potential which, more generally, is a characteristic feature of electrodes consisting of phase separating materials exposed to low currents [1] and [2].
Therefore, in order to ensure a credible SOC estimation in such types of batteries, it is very important to analyze potential influential factors that can bias the voltage level. Whereas the operating temperature of the battery and the applied current rate do bias the voltage level, their influences can for a certain battery type be relatively accurately determined in advanced SOC estimators. In contrast, one of the important phenomena that renders accurate SOC estimation difficult is the memory effect, which is well known in Ni-Cd and Ni-MH batteries. As reported in [3], the memory effect is manifested in a reduced voltage in the discharge curves when the batteries undergo a repeated shallow-depth discharge for a large number of cycles [4] to [7]; under such conditions the discharge voltage seems to memorize the depth of discharge of the previous cycling. This memory effect leads to a reduction in practical cell capacity at a fixed cutoff voltage and/or to a wrong estimate of the state of charge (SOC) of the cell.

In contrast to the memory effect observed in NiMH batteries, the memory effect in Li-ion batteries based on phase separating materials occurs after only one partial charge/discharge cycle [3]. In agreement with [3] the term memory effect denotes abnormal changes in working voltage, which does not include 
hysteresis behavior during charge and discharge. As $\mathrm{LiFePO}_{4}$ (LFP) is a phase separating material characterized by a very flat voltage profile between the spinodal points of the chemical potential, the small memory bump that already appears after only one cycle of partial charge and discharge can lead to substantial miscalculations in estimating the state of SOC by software algorithms.

Sasaki et al. [3] have developed a well-defined procedure to observe and study the memory effect in detail. As demonstrated in Fig. 2, this procedure consists of: a) partially charging the empty battery with moderate currents, b) a first rest period, c) a discharge with moderate currents, d) a second very short rest and e) a memory releasing cycle, during which the memory effect is observed in the vicinity of the terminal SOC of the partial charge in step a). Sasaki et al. have also clearly related this intra-electrode phenomenon to the macroscopic battery output, which has importance for most battery uses. They have shown a strong correlation between the initial SOCs of the memory writing and the releasing cycle and the magnitude of the memory effect. For example, the memory effect is much more pronounced if the partial discharge in the memory writing cycle stops at higher SOC values [3]. In addition, Jia et al. [8] investigated the influence of the rest time after the memory writing cycle on the magnitude of the memory effect and reported that a prolonged relaxation amplified the memory effect.

Farkhondeh et al. [9] were the first to successfully simulate experimental findings published in papers [3] and [8]. They used multi-particle model with the additionally introduced parameter of active particle surface resistance that was Gaussian distributed among particles. Even though their model was able to replicate the memory effect in multi-particle phase separating electrode, it lacks the insight that additionally introduced resistant parameter also corresponds to different particle sizes.

Although Sasaki et al. [3] had been the first to report this effect, it was not until the publication by Kondo et al. [10] that a consistent mechanistic explanation of the memory effect was proposed. They based their analyses on a simplified electrochemical model neglecting mass transport phenomena such as electronic conductivity and lithium diffusion in the solid and liquid phases while assuming a uniform concentration of $\mathrm{Li}$ within the active particles and in the electrode pores. Their model is similar to the one from [9] with the introduction of particle size distribution. They associate the memory effect, i.e. a bump in the voltage profile, with a reduced active particle population that appears as a consequence of a shim shaped Li-composition distribution which, in turn, is a result of particle size distribution. Kondo et al. [10] further state that there is no memory effect if there is no rest after a first partial charge (step b) above). This statement is not fully consistent with their own results shown in the paper where it is clearly seen that this case is also characterized by a reduced active particle population and a bump in the voltage profile. However, the voltage bump occurs at a lower voltage and its magnitude is also slightly lower. This less intense but non-vanishing voltage bump can be explained by a larger active particle population due to the absence of a rest period.

Obviously, the memory effect only arises if the cathode is not fully lithiated during a discharge process (step c) above). Therefore, the memory effect is indeed important in real battery applications, as control algorithms in appliances prevent the battery of being fully discharged. Hence, this phenomenon needs to be further scientifically elucidated to form a solid basis for future research.

As shown above, the existing literature does not consistently reveal the phenomenological background for the onset or absence of the memory effect. The paper at hand, therefore, enriches the literature on the memory effect by providing, for the first time i) a clear experimental and theoretical description of the conditions that lead to the onset or absence of the memory effect and ii) the underlying causal chain of phenomena from the collective particle-by-particle intra-electrode phenomena to macroscopic voltage output of the battery.

\section{METHODS}

To provide a full reasoning of the addressed phenomena this analysis originates from very accurate experimental measurements, which clearly represent the macroscopic battery outputs. This experimental analysis is complemented by a detailed simulation study based on an advanced continuum level model, which reveals the intra-electrode phenomena thus reasoning the causal chain of phenomena and completing the overall analysis.

\subsection{Experimental}

\subsubsection{Active Cathode Materials}

In this study two types of $\mathrm{LiFePO}_{4} / \mathrm{C}$ powder materials were used as active materials for LFP cathodes: a) A commercial powder provided by Targray Group (SLFP02002) further designated as "Targray LFP". 
According to the specifications, this LFP material has a specific surface area of $11 \pm 2 \mathrm{~m}^{2} \mathrm{~g}^{-1}$, an agglomerate size of $3.0 \pm 1.0 \mu \mathrm{m}$ by the D50 criterion, and a native carbon content of $2.0 \pm 0.5$ wt.\%. b) The second type of LFP material (LFP-pcrm) was prepared by a novel pulse combustion reactor method in a slightly reductive environment, as described in detail in our previous paper [11]. Briefly, the material was synthesized in a reactor setup consisting of a Helmholtz-type pulse combustor with a natural frequency of $280 \mathrm{~Hz}$ (at a temperature of around 1250 $\mathrm{K})$ and a $4 \mathrm{~m}$ long stainless steel reactor pipe. Air is supplied to the combustor by way of a blower through an aerodynamic valve. The method allows very precise control of atmosphere in the reactor, as well as the frequency and amplitude of pulses, all of which have a pronounced effect on the reaction outcome [12].

In the present synthesis the spraying gas was 99.9 $\%$ nitrogen with a pressure of 1.5 bar and a flow of 45 $\mathrm{mL} \min ^{-1}$, measured using a thermal mass flow meter. The precursor was composed of $13.5 \mathrm{~g}$ of $\mathrm{LiNO}_{3}, 76.1$ $\mathrm{g}$ of $\mathrm{Fe}\left(\mathrm{NO}_{3}\right)_{3} \cdot 9 \mathrm{H}_{2} \mathrm{O}, 36.4 \mathrm{~g}$ of triethyl phosphate, $78.5 \mathrm{~g}$ of glycine, $30.9 \mathrm{~g}$ of $\mathrm{NH}_{4} \mathrm{NO}_{3}$, everything dissolved in $400 \mathrm{~g}$ of deionized water. We used a 4 mole $\%$ excess of lithium due to losses in the reactor and annealing oven. The temperature at $0.5 \mathrm{~m}$ after the spray nozzle was maintained at $(700 \pm 5){ }^{\circ} \mathrm{C}$ with the amount of precursor sprayed $(20 \pm 1) \mathrm{mLmin}^{-1}$. The frequency of combustion was maintained at $240 \mathrm{~Hz}$. The prepared material was collected in an electrostatic precipitator and annealed in an electrical oven under a constant argon flow and in presence of carbon at $700{ }^{\circ} \mathrm{C}$ for 6 hours. The annealed material had a tap density of approximately $1 \mathrm{gcm}^{-3}$ and was used as prepared for the preparation of cathodes.

\subsubsection{Electrode Composite and Electrode Preparation}

Using the selected $\mathrm{LiFePO}_{4} / \mathrm{C}$ material porous electrodes were prepared by a standard procedure for preparation of laboratory-scale LFP electrodes. We prepared a homogeneous slurry (dispersion) of the solid components in beforehand prepared $15 \mathrm{mg}$ $\mathrm{mL}^{-1}$ solution of PVdF binder (182702 Aldrich) in NMP solvent (99.5\% pure, 8.06072.2500 Merck) to obtain the final (dry) electrode composite composition LFP/C $:$ CB $:$ PVdF $=90: 5: 5$ (wt./wt.). The slurry was homogenized in a planetary mill for $30 \mathrm{~min}$ at $300 \mathrm{rpm}$. The slurry was applied to the surface of carbon coated Al foil by using automated doctorblade applicator (the distance between the blade and the surface of $\mathrm{Al}$ foil was set to $200 \mu \mathrm{m})$. The
Al foil with coating was in the first step dried for 3 $\mathrm{h}$ at $90{ }^{\circ} \mathrm{C}$ at reduced pressure (10 mbar absolute). After that we cut out circular electrodes with $16 \mathrm{~mm}$ diameter (geometric area $\mathrm{A}=2.01 \mathrm{~cm}^{2}$ ). We pressed the electrodes by applying $5 \mathrm{t}\left(2.5\right.$ tons per $\left.1 \mathrm{~cm}^{2}\right)$ for $1 \mathrm{~min}$ in a hydraulic press. Before transferring in an argon-filled glove box the electrodes were dried under vacuum overnight at $90{ }^{\circ} \mathrm{C}$ in vacuum chamber. This means the prepared electrodes had LFP mass loadings of about $3 \mathrm{mg}$ per $\mathrm{cm}^{2}$ of the electrode geometric area (composite loading $3.3 \mathrm{mg}$ per $\mathrm{cm}^{2}$ ) and the obtained composite thickness was in the range 18 to $19 \mu \mathrm{m}$. We calculated the electrode porosity by taking the values of electrode composite mass, thickness and the bulk densities of the composite components to be 42 vol. $\%$; which is comparable with the typical range found for LFP cathodes with good performance [13] and [14].

\subsubsection{Cell Preparation and Electrochemical Measurements}

All the electrochemical experiments were conducted by using vacuum sealed pouch-type cells. The conventional 2-electrode $\mathrm{LiFePO}_{4}-\mathrm{Li}$ cells were assembled in an Ar-filled glove box by using metallic lithium foil (geometric area of $2.5 \mathrm{~cm}^{2}$ ) as a counter electrode. As a separator, a glass fiber filter paper (Whatman, glass microfiber) was used. The electrolyte used was commercialized "LP-40", 1 M solution of $\mathrm{LiPF}_{6}$ in ethylene carbonate/diethyl carbonate $(\mathrm{EC}: \mathrm{DEC}=1: 1 \mathrm{wt} / \mathrm{wt}$, Merck$)$.

Standard chronopotentiometric (galvanostatic) measurements and all the memory effect experiments were performed using a "VPM3" (Bio-Logic) potentiostat/galvanostat running with $\mathrm{EC}-\mathrm{Lab}{ }^{\circledR}$ software. All the cells were electrochemically precycled $(5$ cycles $\mathrm{C} / 10)$ in the voltage window $2.5 \mathrm{~V}$ to $4.1 \mathrm{~V}$ before testing for the memory effect. It is important to note that in order to diminish the effects of surrounding temperature variation, all the measurements were performed by keeping battery cell temperature constant in a thermostatic bath (dipped in silicone oil with temperature $25 \pm 0.1{ }^{\circ} \mathrm{C}$ ).

\subsection{Simulation Model}

Simulation analysis relies on an advanced continuum Li-ion battery modeling framework [15] featuring sequential multi-scale model linking [16]. This is achieved by thermodynamically consistent upscaling [17] of an advanced and detailed model of LFP particle potential as a function of lithiation determined by a thermodynamically consistent derivation [18]. Another merit of the advanced continuum 
modelling framework arises from a more consistent reproduction of real multi-particle size distributions and particle connectivity and, therefore, electrode topology on the level of the entire electrode. This consistent transfer of a fundamental understanding of materials to the cell level and modeling of all relevant phenomena influencing battery performance on the continuum scale enhances the prediction capability of the proposed modeling framework in analyzing the memory effect compared to previous works [3], [9] and [10].

\subsubsection{Constitutive Equations}

The functioning of battery cells used in this study is described using Newman's approach [19], where electrochemical and transport phenomena are described by four partial differential equations for charge and mass conservation in the solid and the electrolyte (Eqs. (1) to (4)):

$$
\begin{gathered}
\frac{\partial \varepsilon c_{e}}{\partial t}=\nabla \cdot\left(D_{e}^{e f f} \nabla c_{e}\right)+\left(1-t_{+}\right) a_{p} j_{B V}, \\
\nabla \cdot\left(\kappa^{e f f} \nabla \phi_{e}-\frac{2 R T \kappa^{e f f}}{F}\left(1-t_{+}\right) \nabla \ln c_{e}\right)=F a_{p} j_{B V}, \\
\nabla \cdot\left(\sigma^{e f f} \nabla \phi_{s}\right)=-F a_{p} j_{B V}, \\
\frac{\partial c_{s}}{\partial t}=\nabla \cdot\left(\frac{D_{s} c_{s}}{R T} \nabla \mu_{s}\right) .
\end{gathered}
$$

The same boundary conditions are used as in reference [20].

The intercalation current across the cathode active particle surface was modeled with the ButlerVolmer equation:

$$
j_{B V}=\frac{i_{0}}{F}\left[\exp \left(-\frac{\alpha F}{R T} \eta\right)-\exp \left(\frac{(1-\alpha) F}{R T} \eta\right)\right] .
$$

Nomenclature from Eqs. (1) to (5) is summarized in the Section 6 at the end of the paper.

For the chemical potential of active particles, the zero dimensional regular solution model was used as described in reference [17]. It has a shape of a spinodal curve at high overpotentials and a plateau-like shape at low overpotentials (Fig. 1).

Such a shape of the potential ensures a consistent description of intra-particle phase separation [18] and [21] to [28] as well as inter particle lithium exchange first modeled by Dreyer et al. [29].

In addition to the model by Dreyer et al [29], particle agglomeration and crystallographic orientations were taken into account by introduction of connectivity [30] and [31] and permeability [15] parameters that govern inter particle lithium exchange.

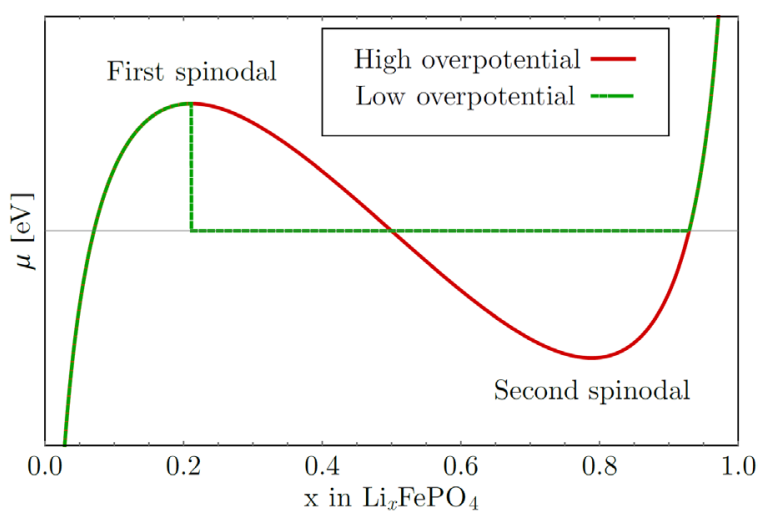

Fig. 1. Spinodal shaped chemical potential $\mu$ dependency on particle state of charge ( $x$ in $\mathrm{Li}_{x} \mathrm{FePO}_{4}$ ) at charge for the case of high and low overpotential curve

Intercalation rate dependency on the particle size was introduced to the model by a variable regular solution parameter as described in [17], which was crucial for plausibly simulating the memory effect. This is in agreement with Kondo et al. [10] who showed that the memory effect is a consequence of the shorter characteristic intercalation times for smaller particles. Log-normal particle size distribution was used based on scanning and transmission electron microscopy measurements.

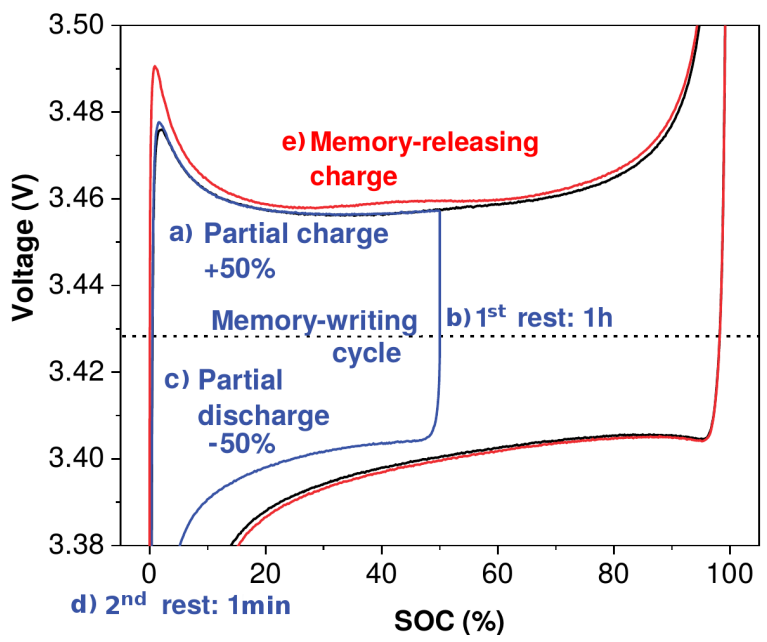

Fig. 2. Memory effect measured by the same protocol as used by Sasaki et al. [3]; blue line represents the memory writing cycle, red line is the memory releasing cycle and black line represents baseline discharge curve. Individual parts of the protocol chronologically follow: a) partial charge, b) 1 st rest, c) partial discharge, d) $2^{\text {nd }}$ rest and e) memory releasing cycle 
All the simulations presented in the Results section were performed on a half cell with a phase separating multi-particle cathode material (LPF), separator and lithium metal anode.

\section{RESULTS}

Three distinctive experiments were made, that were later simulated with the model presented in section 1.2. The combined results of experiment and simulation offer new insights into the mechanism of memory effect.

\subsection{Experimental Results}

The experimental results presented in Fig. 2 were obtained by following exactly the same experiment procedure as proposed in the pioneering work of Sasaki et al. [3]. In agreement with the procedure presented in the Introduction, a battery cell was charged to half of its maximal capacity, left to rest for an hour (first rest) and then discharged back. This first half cycle is called the memory writing cycle [3]. After the battery had been fully charged, a memory releasing cycle was initiated. This cycle is characterized by a small bump in the voltage curve, called the memory effect [3]. Note that the memory effect appears in the vicinity of the SOC value of the first rest in the preceding memory writing cycle and should not been mistaken for the first, bigger bump seen in all curves at low SOCs.

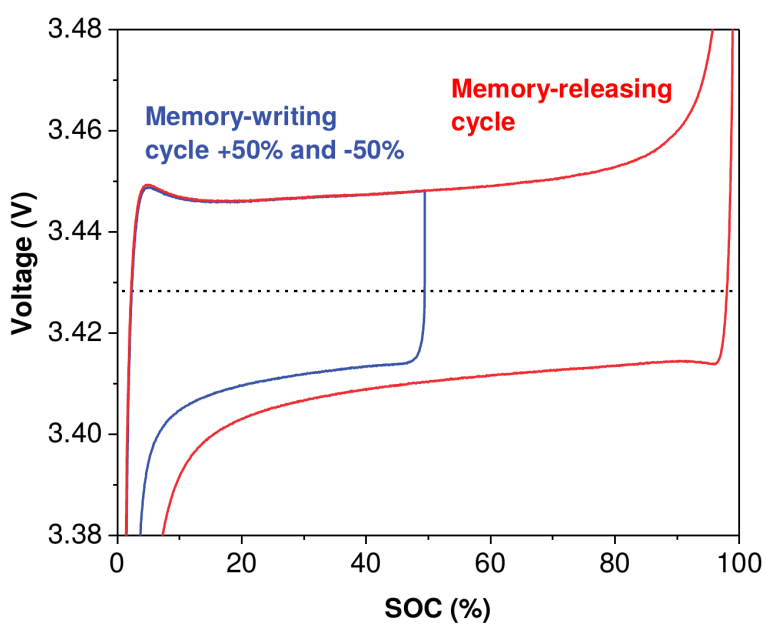

Fig. 3. A memory effect experiment executed using the same protocol as in Fig 2, but to the cut off voltage of $2.8 \mathrm{~V}$ at the end of the memory writing cycle

Fig. 3 shows experimental result where the cut off voltage of memory writing cycle was lowered to $2.8 \mathrm{~V}$. The results clearly indicate that the memory effect is absent if the cut off voltage in the memory writing cycle is sufficiently low thus erasing the memory effect, which was, based on simulation results, to a certain degree already discussed in paper of Farkhondeh et al. [9].

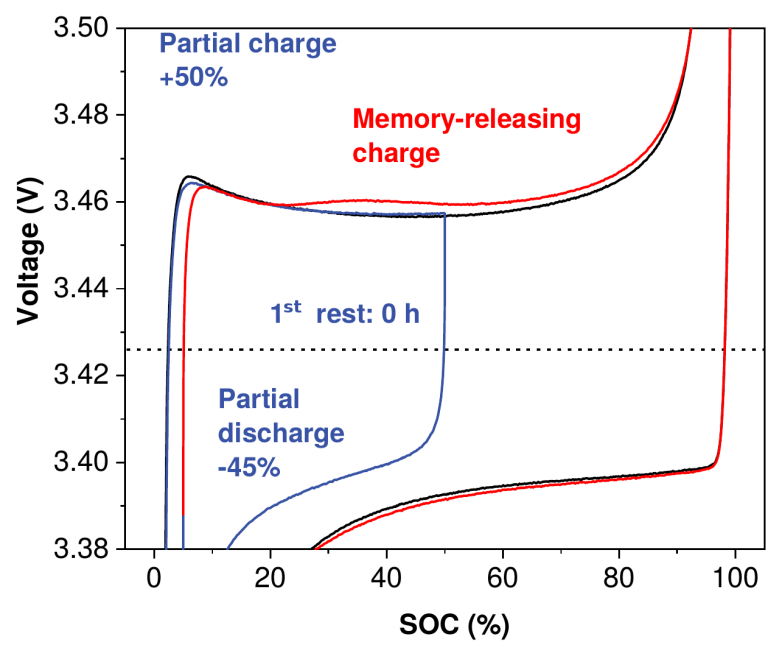

Fig. 4. A memory effect experiment executed using the same protocol as in Fig. 2, but without first rest in the memory writing cycle

Fig. 4 shows experimental evidence that the memory effect also exists in the limiting case where the time of the first rest in the memory writing cycle is reduced to zero. This has not been shown until now. In fact, an opposite claim was reported in [10], which however was not consistently supported by experimental and simulation evidence.

Figs. 2, 3 and 4 provide, for the first time, a consistent experimental detection of the onset or absence of the memory effect in phase separating Liion battery cell cathode materials at various conditions of interest. More detailed discussion of these results is given in the Discussion section in combination with the simulation results.

\subsection{Simulation Results}

To ensure clear causal relations from macroscopic experimental parameters to the simulated phenomena on the particle level, simulations performed by the model described in Section 1.2 were replicated for exactly the same scenarios as used in the experimental tests. Fig. 5, which presents results according to the scenario proposed by Sasaki et al. [3] (i.e. experimental results in Fig. 2) confirms the capability of the model to credibly replicate and predict the memory effect. It is for the first time that a fully coupled Newman-based continuum model was used for successful simulation 


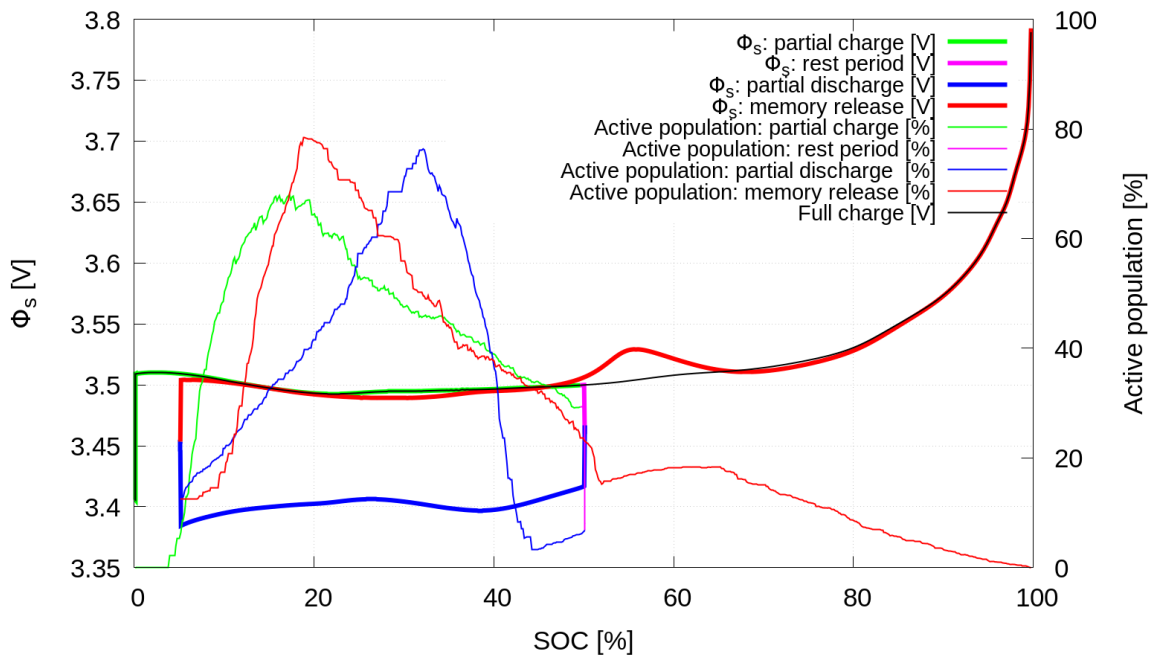

Fig. 5. Simulation of memory effect calculated for the protocol presented in Fig. 2, with the model described in Section 1.2
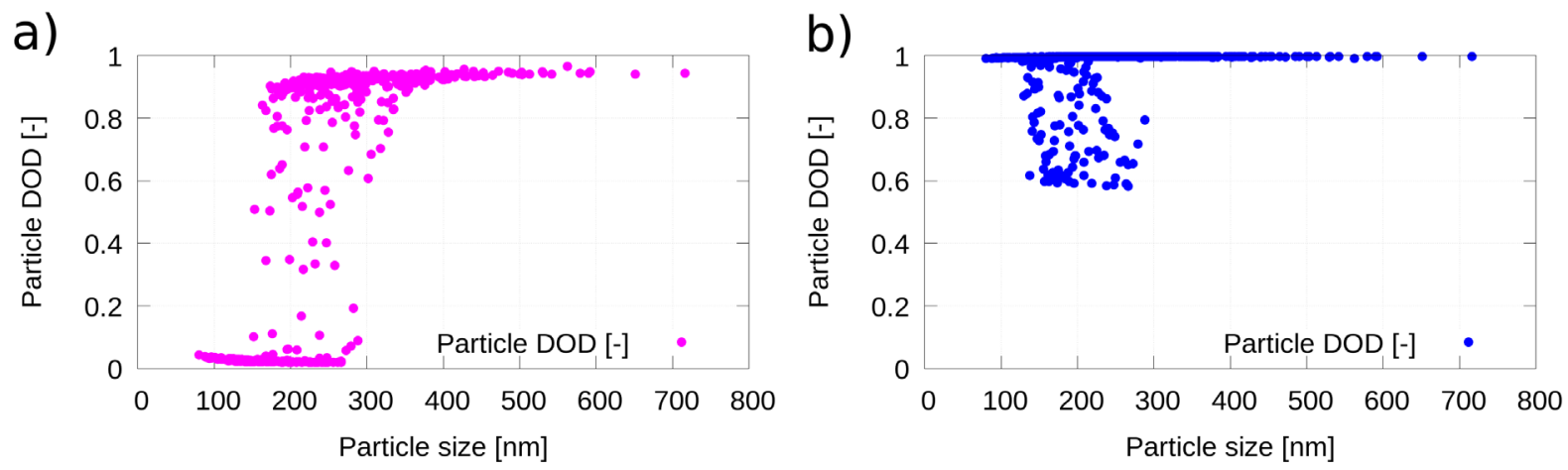

Fig. 6. Simulation of inter-particle lithium distribution in the cathode, during a) first rest and b) second rest corresponding to the protocol presented in Fig. 5

of the memory effect, which was made possible by innovative extensions presented in Section 1.2. The plausibility of the applied model is demonstrated in Fig. 6, where the average lithium concentration inside a particle (Particle DOD) is plotted versus particle size for particles in the cathode at two distinctive time instants of the memory writing protocol: at the end of first rest and at the end of the memory writing cycle (as presented in Figs. 2 to 4). Fig. 6b clearly presents the so called shim shaped particle distribution at the end of second rest, which is a prerequisite for the observation of the memory efect [10].

Likewise, Fig. 7 shows simulation results for the case with low cut-off voltages at the end of the memory writing cycle. Again, it can be concluded that the simulation results are in full agreement with the experimental observations presented in Fig. 3, as both cases predict memory erasure due to full particle lithiation at the end of memory writing cycle. This is clearly confirmed in Fig. 8 showing particle DOD vs. particle size, where all particles are lithiated due to a low cut-off voltage thus erasing the memory effect due to absence of the shim shaped particle distribution at the end of the second rest.

Fig. 9 serves as simulation support to findings from Fig. 4, where the memory effect was observed even at the zero first rest and Fig. 10 shows the corresponding particle DOD vs. particle size.

All the simulation results coincide very well with the experimental results, thus presenting a strong joint platform for several new insights into the mechanism of memory effect, as explained in the Discussion section.

\section{DISCUSSION}

The result in Fig. 2 proves the reproducibility of the memory effect and serves as a calibration for the 


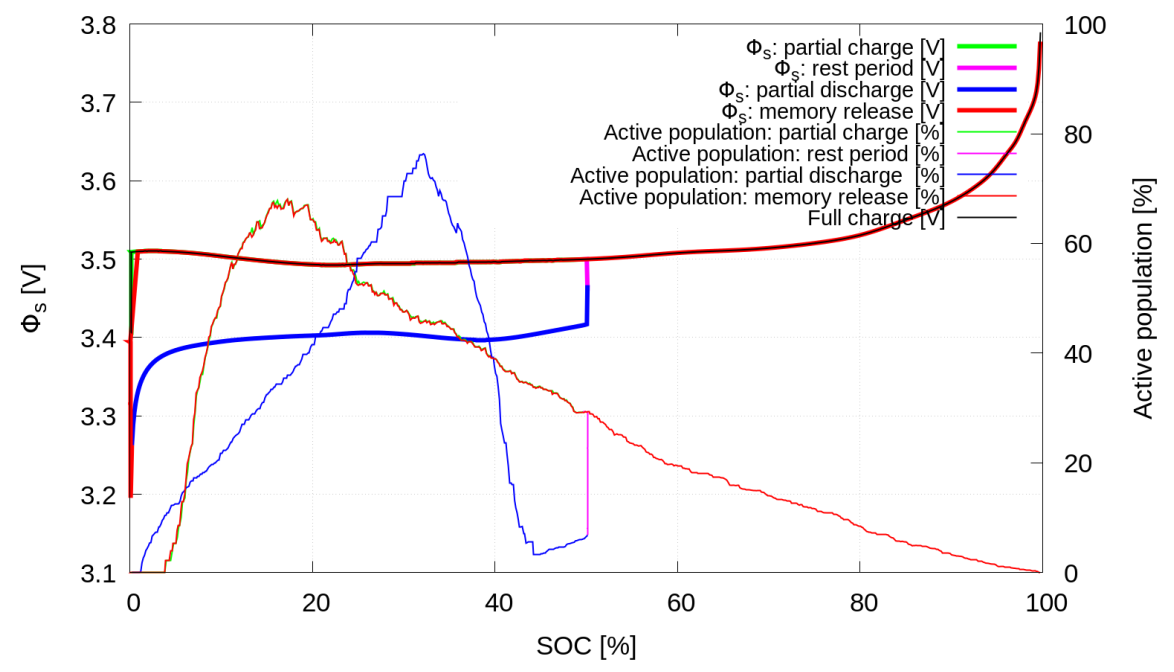

Fig. 7. Simulation of memory effect calculated for the same protocol as in Fig. 5 but with the cut of voltage at the end of the memory writing cycle lowered to $2.8 \mathrm{~V}$. No memory effect is observed in this case

a)

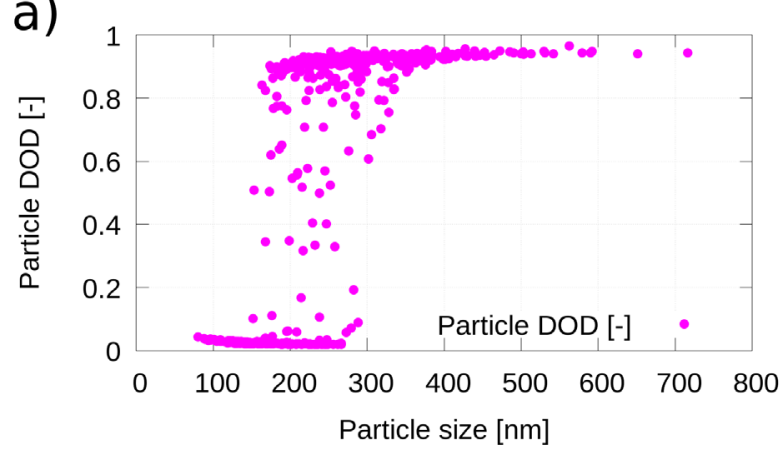

b)

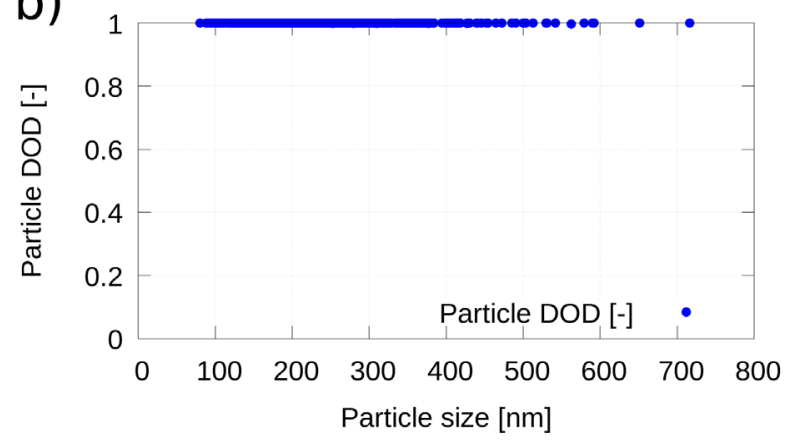

Fig. 8. Simulation of inter-particle lithium distribution in the cathode, during a) first rest and b) second rest corresponding to the protocol presented in Fig. 7

model, whereas the simulation result in Fig. 5 proves that a consistent continuum-level model of the full half-cell is capable of simulating the memory effect. From Fig. 6 it can be seen that the kinetics in smaller particles is faster in comparison to larger particles and the smaller particles are therefore depleted of lithium during the memory writing cycle, whereas larger particles remain full of lithium (Fig. 6a). It is worth noting that in Fig. 6 the division between lithiated and delithiated particles is not as sharp as schematically presented in [10]. This is reasoned by the fact that, unlike in [10], a full half-cell model capable of considering variation of all electrode relevant parameters was used in the present study. Therefore, nonuniform concentration and potential fields partially compete with size dependent particle kinetics leading to a less sharp division between lithiated and delithiated particles.
As a result of this particle size dependent kinetics, the electrode becomes inter-particle phase separated at the end of the first rest, with a small portion of particles with a size of around $200 \mathrm{~nm}$ still being active (Fig. 6a). After the discharge part of the memory writing cycle, the faster kinetics of smaller particles favors their faster lithiation, as presented in Fig. 6b. As a result, only medium-sized particles remain partially delithiated (remain active). The same effect is also seen in Fig. 5, where the active particle population does not drop to zero at the second rest, i.e. between partial discharge and memory release. Resultantly, the so called shim shaped particle distribution is formed as presented in Fig 6b, which is quantitatively identical to the one calculated by Kondo et al. [10]. This aligns the present results with the ones shown in [10], confirming the necessity of the shim shaped particle distribution to generate the 


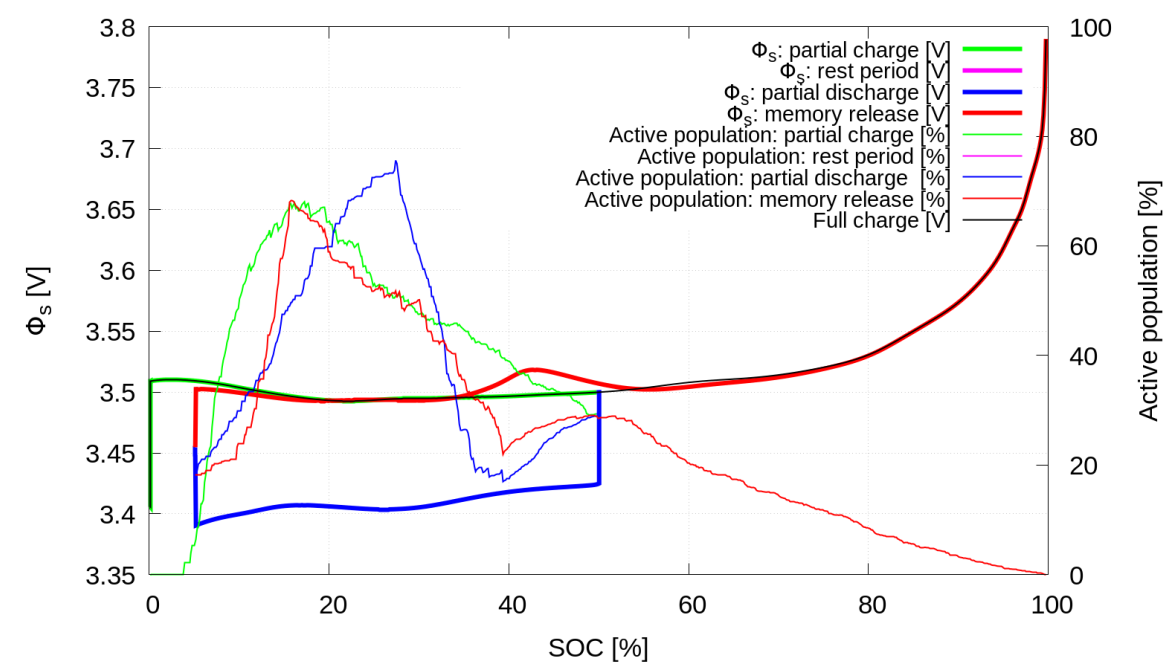

Fig. 9. Simulation of memory effect calculated for the same protocol as in Fig. 5 but without fist rest
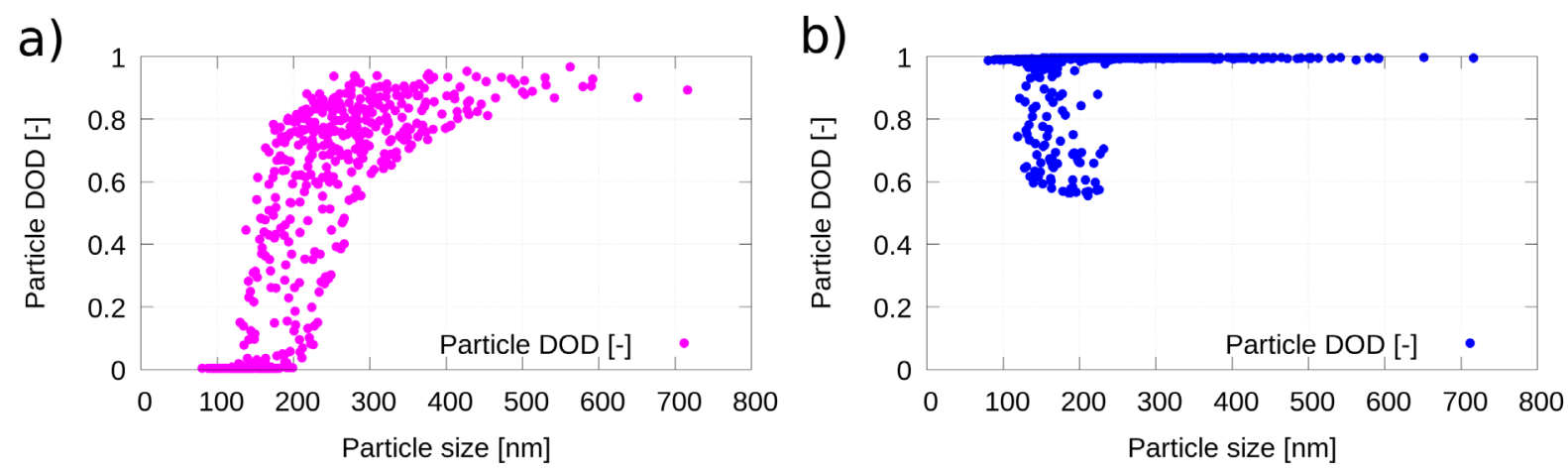

Fig. 10. Simulation of inter-particle lithium distribution in the cathode, during a) first rest and b) second rest corresponding to the protocol presented in Fig. 9

memory effect. Kondo et al. [10] further supported this statement with the need of a short second rest period that prevents relaxation of this shim shaped particle distribution.

In the subsequent memory releasing cycle a sharp drop in active particle population can be observed in the vicinity of the SOC value of the first rest in the preceding memory writing cycle, i.e. a SOC value of around $50 \%$. This observation, which is in-line with the one provided by [10], is reasoned by the fact that the active particle population drops when the lithiation level of the electrode approaches values at which partially delithiated particles in the shim shaped particle distribution become active. This effect is certainly observable only at sufficiently moderate currents that favor inter-particle phase separation.

Comparison of the results shown in Fig. 3 and Fig. 7 clarifies the speculations about the cases where memory effect was not observed [8] and [9]. Namely, the memory effect can be erased by simply sufficiently lowering the cut-off voltage $(2.8 \mathrm{~V})$ at the end of the writing cycle. The same experimental result was also achieved by a longer voltage hold at cut-off voltage values up to $3.3 \mathrm{~V}$ at the end of the memory writing cycle.

The main precondition for the onset of the memory effect is, as analyzed earlier, the presence of the shim shaped particle distribution at the end of the second rest. This inherently implies that the electrode is not fully lithiated. A logical measure for erasing the memory effect is thus a full lithiation of the electrode, which can be achieved by any of the previously proposed methods: a very low cut-off voltage or a longer voltage hold at a cut-off voltage below the voltage of the lower spinodal (Fig. 1). Thus, all the particles are driven to the regime of high concentrations (above the second spinodal point (Fig. 1), where the chemical potential dependency on 
concentration is a monotonous function). Inter particle phase separation of the system in such a regime is, therefore, not possible and all the particles occupy equal state of charge at the same value of the chemical potential leading to the absence of the shim shaped particle distribution. This can clearly be seen in Fig. $8 \mathrm{~b}$ and from the active particle population curve in Fig. 7, which indicates zero active particle population at the end of second rest. These observations are in line with the model of Dreyer et al. [29], Farkhondeh et al. [9] and also with the model of Kondo et al. [10], however until now the entire causal chain of phenomena from the macroscopic voltage output of the battery to collective particle-by-particle intraelectrode phenomena was not elucidated to that extent.

In short, the presented results provide a clear confirmation that the memory effect can only be observed if a battery cell is not fully discharged (discharged to low cut off voltages) in the memory writing cycle. Despite this important fact, addressing the influence of the memory effect in appliances equipped with Li-ion batteries composed of phase separating materials is still important, as control algorithms prevent the battery from being fully discharged.

The combined findings displayed in Figs. 4, 9 and 10 provide the basis for explaining another intriguing fact about the memory effect. Fig. 4 shows experimental evidence that the memory effect also exists in the limiting case where the first rest period in the memory writing cycle is reduced to zero. This result opposes the measurements by Jia et al. [8], where the absence of the memory effect at the zero first rest experiment can be attributed to the above average connectivity of active particles in the cathode, that prevents establishment of shim shaped interparticle lithium distribution after memory writing cycle. Likewise, this opposes the statement published in [10] that the memory effect is not present in the absence of the first rest, although the simulation results in [10] do not fully support this statement, as they also predict a drop of active particle population also in this case.

The presence of the memory effect in the case of zero first rest period can be explained by the fact that, even though the particles are not relaxed after the partial charge part of the memory writing cycle, a wide distribution of particles across the full range of concentrations can be observed (Fig. 10a). Qualitatively this distribution, where smaller particles are delithiated and larger particles remain lithiated (Fig. 10a), looks similar to the one presented in Fig. 6a. The two distributions differ quantitatively as expected. In the case with an absent first rest, the active particle population is larger. However, upon subsequent lithiation, the faster kinetics of smaller particles in combination with all particles not being fully lithiated again forms a shim shape particle distribution at the end of the second rest (Fig. 10b). This provides a reasoning for the experimental observation of the memory effect when the first rest period is zero, which is well aligned with the simulation results. The latter again predict a decrease of active particle population, which is associated with an increase in charging voltage as presented in Fig. 9.

\section{CONCLUSIONS}

The memory effect previously observed in several Li-ion phase separating multi-particle cathodes was investigated by a rigorous experimental and an advanced simulation approach. The good agreement between the simulated and measured results offered several new insights in the mechanistic origin of the memory effect. Experiment and simulation of low cut off voltage and the absence of a first rest in the memory writing cycle are shown as two previously insufficiently explained cases. It was shown that the memory effect is erased upon lithiation of the electrode after the memory writing cycle, which can be achieved by either a very low cut-off voltage or a longer voltage hold at a cut-off voltage below the voltage of the lower spinodal. In addition, it was shown that the absence of the first rest in the memory writing cycle itself is not a sufficient condition for erasing the memory effect.

\section{ACKNOWLEDGEMENTS}

The authors acknowledge financial support from the Slovenian Research Agency (research core funding No. P2-0401 and P2-0393 and project J7-8270). This project has received funding from the European Union's Horizon 2020 research and innovation programme under grant agreement No 769506 .

\section{NOMENCLATURES}

$a_{p} \quad$ volumetric specific active, $\left[\mathrm{m}^{-1}\right]$

$\alpha$ particle surface area charge transfer coefficient, $[-]$

$c_{e}$ electrolyte $\mathrm{Li}$ concentration, $\left[\mathrm{mol} / \mathrm{m}^{3}\right]$

$c_{s}$ solid phase Li concentration, $\left[\mathrm{mol} / \mathrm{m}^{3}\right]$

$D_{e}^{e f f}$ effective diffusion coefficient in electrolyte, $\left[\mathrm{m}^{2} / \mathrm{s}\right]$

$D_{s}$ diffusion coefficient in solid phase, $\left[\mathrm{m}^{2} / \mathrm{s}\right]$ 
$\varepsilon \quad$ porosity of electrode, [-]

$\eta$ overpotential, [V]

$F \quad$ Faraday constant, [As/mol]

$\phi_{e}$ electrolyte potential, [V]

$i_{0} \quad$ Butler-Volmer exchange current density, $\left[\mathrm{A} / \mathrm{m}^{2}\right]$

$j_{B V}$ Butler-Volmer intercalation current density, $\left[\mathrm{A} / \mathrm{m}^{2}\right]$

$k^{e f f}$ electrolyte effective ionic conductivity, [S/m]

$\mu_{s} \quad$ solid phase chemical potential, $[\mathrm{eV} / \mathrm{mol}]$

$\sigma^{\text {eff }}$ solid phase effective electric conductivity, $[\mathrm{S} / \mathrm{m}]$

$R$ gas constant, $[\mathrm{J} /(\mathrm{molK})]$

$T$ temperature, $[\mathrm{K}]$

$t$ time, [s]

$t_{+}$transference number, [-]

\section{REFERENCES}

[1] Ellis, B.L., Lee, K.T., Nazar, L.F. (2009). Positive electrode materials for Li-lon and Li-batteries. Chemistry of Materials Review, vol. 22, p. 691-714, D0l:10.1021/cm902696j.

[2] Padhi, A.K., Nanjundaswamy, K.S., Goodenough, J.B. (1997). Phospho-olivines as positive-electrode materials for rechargeable lithium batteries. Journal of the Electrochemical Society, vol. 144, no. 4, p. 1188-1194, DOI:10.1149/1.1837571.

[3] Sasaki, T., Ukyo, Y., Novák, P. (2013). Memory effect in a lithium-ion battery. Nature Materials, vol. 12, p. 569-575, DOI:10.1038/nmat3623.

[4] Davolio, G., Soragni, E. (1998). The memory effect on nickel oxide electrodes: Electrochemical and mechanical aspects. Journal of Applied Electrochemistry, vol. 28, no. 12, p. 13131319, DOI:10.1023/A:1003452327919.

[5] Huggins, R.A. (2006). Mechanism of the memory effect in "Nickel" electrodes. Solid State Ionics, vol. 177, no. 26-32, p. 2643-2646, Dol:10.1016/j.ssi.2006.03.005.

[6] Sato, Y., Takeuchi, S., Kobayakawa, K. (2001). Cause of the memory effect observed in alkaline secondary batteries using nickel electrode. Journal of Power Sources, vol. 93, no. 1-2, p. 20-24, DOI:10.1016/S0378-7753(00)00506-1.

[7] Barnard, R., Crickmore, G.T., Lee, J.A., Tye, F. (1980). The cause of residual capacity in nickel oxyhydroxide electrodes. Journal of Applied Electrochemistry, vol. 10, no. 1, p. 61-70, DOI:10.1007/BF00937339.

[8] Jia, J., Tan, C., Liu, M., Li, D., Chen, Y. (2017). RelaxationInduced Memory Effect of $\mathrm{LiFePO}_{4}$ Electrodes in Li-lon Batteries. ACS Applied Materials \& Interfaces, vol. 9, p. 24561-24567, D0I:10.1021/acsami.7b05852.

[9] Farkhondeh M., Pritzker M., Fowler M., Safari M. and Delacourt C. ( 2014). Mesoscopic modeling of Li insertion in phase-separating electrode materials: application to lithium iron phosphate. Physical Chemistry Chemical Physics, vol. 16, no. 41, p. 22555-22565, D0l:10.1039/C4CP03530E.

[10] Kondo, H., Sasaki, T., Barai, P., Srinivasan, V. (2018). Comprehensive study of the polarization behavior of $\mathrm{LiFePO}_{4}$ electrodes based on a many-particle model. Journal of The Electrochemical Society, vol. 165, p. A2047-A2057, DOI:10.1149/2.0181810jes.
[11] Križan, G., Križan, J., Dominko, R., Gaberšek, M. (2017). Pulse combustion reactor as a fast and scalable synthetic method for preparation of Li-ion cathode materials. Journal of Power Sources, vol. 363, pp. 218-226, D0l:10.1016/j. jpowsour.2017.07.083.

[12] Križan, G., Križan, J., Bajsić, I., Gaberšček, M. (2018). Control of a pulse combustion reactor with thermoacoustic phenomena. Instrumentation Science and Technology, vol. 46, no. 1, p. 43-57, DOI:10.1080/10739149.2017.1320288.

[13] Fongy, C., Gaillot, A.-C., Jouanneau, S., Guyomard, D., Lestriez, B. (2010). Ionic vs electronic power limitations and analysis of the fraction of wired grains in $\mathrm{LiFePO}_{4}$ composite electrodes. Journal of The Electrochemical Society, vol. 157, no. 7, p. A885-A891, Dol:10.1149/1.3432559.

[14] Fongy, C., Jouanneau, S., Guyomard, D., Badot, J., Lestriez, B. (2010). Electronic and ionic wirings versus the insertion reaction contributions to the polarization in $\mathrm{LiFePO}_{4}$ composite electrodes. Journal of the Electrochemical Society, vol. 157, no. 12, p. A1347-A1353, D0I:10.1149/1.3497353.

[15] Katrašnik, T., Mele, I., Zelič, K. (2019). advanced continuum Li-ion battery modelling framework. 11 $1^{\text {th }}$ International Conference on Applied Energy, Västerås.

[16] Franco, A.A., Rucci, A., Brandell, D., Frayret, C., Gaberscek, M., Jankowski, P., Johansson, P. (2019). Boosting rechargeable batteries R\&D by multiscale modeling: Myth or reality? Chemical Reviews, vol. 119, p. 4569-4627, D0I:10.1021/acs. chemrev.8b00239.

[17] Zelič, K., Katrašnik, T. (2019). Thermodynamically consistent and computationally efficient OD lithium intercalation model of a phase separating cathode particle. Journal of the Electrochemical Society, vol. 166, p. A3242-A3249, DOI:10.1149/2.0381914jes.

[18] Zelič, K., Katrašnik, T. (2019). Thermodynamically consistent derivation of chemical potential of a battery solid particle from the regular solution theory applied to $\mathrm{LiFePO}_{4}$, Nature Scientific Reports, vol. 9, D0l:10.1038/s41598-019-38635-2.

[19] Newman, J., Tiedemann, W. (1975). Porous-electrode theory with battery applications. AIChE Journal, vol. 21, p. 25-41, DOI:10.1002/aic.690210103.

[20] Colclasure, A.M., Kee, R.J. (2010). Thermodynamically consistent modeling of elementary electrochemistry in lithiumion batteries. Electrochimica Acta, vol. 55, p. 8960-8973, DOI:10.1016/j.electacta.2010.08.018.

[21] Bai, P., Cogswell, D.A., Bazant, M.Z. (2011). Suppression of phase separation in $\mathrm{LiFePO}_{4}$ nanoparticles during battery discharge. Nano Letters, vol. 11, p. 4890-4896, D0l:10.1021/ nl202764f.

[22] Bazant, M.Z. (2013). Theory of chemical kinetics and charge transfer based on nonequilibrium thermodynamics. Accounts of Chemical Research, vol. 46, p. 1144-1160, D0l:10.1021/ ar300145c.

[23] Singh, G.K., Ceder, G., Bazant, M.Z. (2008). Intercalation dynamics in rechargeable battery materials: General theory and phase-transformation waves in $\mathrm{LiFePO}_{4}$. Electrochimica Acta, vol. 53, p. 7599-7613, Dol:10.1016/j. electacta.2008.03.083.

[24] Kao, Y.H., Tang, M., Meethong, N., Bai, J., Carter, W.C., Chiang, Y.M. (2010). Overpotential-dependent phase transformation 
pathways in lithium iron phosphate battery electrodes. Chemistry of Materials, vol. 22, p. 5845-5855, D0I:10.1021/ cm101698b.

[25] Cogswell, D.A., Bazant, M.Z. (2012). Coherency strain and the kinetics of phase separation in $\mathrm{LiFePO}_{4}$ nanoparticles. ACS Nano, vol. 6, p. 2215-2225, Dol:10.1021/nn204177u.

[26] Zeng, Y., Bazant, M.Z. (2013). Cahn-Hilliard reaction model for isotropic Li-ion battery particles. MRS Proceedings, vol. 1542, p. 13-15, Dol:10.1557/opl.2013.740.

[27] Tang, M., Huang, H.Y., Meethong, N., Kao, Y.H., Carter, W.C., Chiang, Y.M. (2009). Model for the particle size, overpotential, and strain dependence of phase transition pathways in storage electrodes: application to nanoscale olivines. Chemistry of Materials, vol. 21, p. 1557-1571, D0l:10.1021/ cm803172s.

[28] Dargaville, S., Farrell, T.W. (2013). The persistence of phaseseparation in $\mathrm{LiFePO}_{4}$ with two-dimensional $\mathrm{Li}+$ transport:
The Cahn-Hilliard-reaction equation and the role of defects. Electrochimica Acta, vol. 94, p. 143-158, D0l:10.1016/j. electacta.2013.01.082.

[29] Dreyer, W., Jamnik, J., Guhlke, C., Huth, R., Moškon, J., Gaberšček, M. (2010). The thermodynamic origin of hysteresis in insertion batteries. Nature Materials, vol. 9, p. 448-453, DOI:10.1038/nmat2730.

[30] Orvananos, B., Ferguson, T.R., Yu, H.C., Bazant, M.Z., Thornton, K. (2014). Particle-level modeling of the charge-discharge behavior of nanoparticulate phase-separating Li-ion battery electrodes. Journal of The Electrochemical Society, vol. 161, p. A535-A546, DOl:10.1149/2.024404jes.

[31] Orvananos, B., Malik, R., Yu, H.C., Abdellahi, A., Grey, C.P., Ceder, G., Thornton, K. (2014). Architecture dependence on the dynamics of nano-LiFePO 4 electrodes. Electrochimica Acta, vol. 137, p. 245-257, D0I:10.1016/j.electacta.2014.06.029. 